\title{
MAGNETOTRANSPORT AND MAGNETIC PROPERTIES OF (Ga,Mn)As AND ITS HETEROSTRUCTURES
}

\author{
H. OHNO \\ Laboratory for Electronic Intelligent Systems \\ Research Institute of Electrical Communication \\ Tohoku University, Katahira 2-1-1, Sendai 980-8577, Japan
}

\begin{abstract}
Introduction of high density of $\mathrm{Mn}$ in $\mathrm{GaAs}$ by low temperature molecular beam epitaxy results in a homogeneous diluted magnetic semiconductor $(\mathrm{Ga}, \mathrm{Mn}) \mathrm{As}$, which exhibits ferromagnetism at low temperatures. Temperature and magnetic field dependence of magnetotransport and magnetization of $(\mathrm{Ga}, \mathrm{Mn})$ As films revealed the Curie temperature $T_{\mathrm{C}}$ which can be as high as $110 \mathrm{~K}$ and the $p-d$ exchange, which explains $T_{\mathrm{C}}$ in the framework of the RKKY interaction. Multilayer heterostructures such as all-semiconductor ferromagnet/nonmagnet/ferromagnet trilayer structures and resonant tunneling diodes have been fabricated and studied. These heterostructure results show the potential of the present material system for exploring new physics and for developing new functionality toward future electronic and optical devices.
\end{abstract}

PACS numbers: 73.61.Ey, 75.50.Pp, 75.70.Cn

\section{Introduction}

Coexistence of ferromagnetism and semiconducting properties in Eu chalcogenides and semiconducting spinels opened rich fields of magnetic cooperative phenomena in semiconductors [1]. In these magnetic semiconductors, the exchange interaction between band electrons and electrons localized at the magnetic elements was shown to play an important and essential role in determining their peculiar and interesting properties. The crystal growth of those compounds, however, was very difficult and heterostructures based on them were prohibitingly difficult to realize. On the other hand, diluted magnetic semiconductors (DMSs), alloys of non-magnetic semiconductor and magnetic elements, have offered a wide variety of materials and structures to further explore the effect of exchange interaction in semiconductors. Study of DMSs and its heterostructures was centered mostly on II-VI based DMSs, where the valence of group II cations is identical to that of the common magnetic ions such as Mn [2] making them relatively easy to prepare in its bulk form as well as in epitaxial form. Difficulty in doping of II-VI based 
DMSs to $p$ - and $n$-type, however, made the transport studies of II-VI based DMSs rather limited, and made the material less attractive for the application purposes. Parenthetically, this situation is changing as the advance in doping technology very recently resulted in observation of carrier mediated ferromagnetism in II-VI DMS $\left(T_{\mathrm{C}}<2 \mathrm{~K}\right)$ [3]. Understanding of carrier induced ferromagnetism in semiconductors was put forward by the study of ferromagnetism in IV-VI DMSs such as PbSnMnTe [4]. IV-VI DMSs and its heterostructures are again not easy to prepare and few study of ferromagnetism in semiconductor heterostructure system has been reported.

The study on III-V based DMSs was initiated to bridge the field of semiconductor technology and the field of magnetic technology. III-V based DMSs not only will enrich the III-V field by introducing a variety of cooperative phenomena via spin-exchange interaction not present in the conventional non-magnetic III-Vs, but also it will allow access of the DMS field to the optical and electrical devices already established in the III-V area. The use of low-temperature molecular beam epitaxy (MBE) resulted in successful epitaxial growth of uniform (In,Mn)As films on GaAs substrates [5]. The detailed magnetotransport study of $p$-type (In,Mn)As films revealed the presence of carrier-induced partial-ferromagnetic ordering at low temperatures [6]. In 1996, our research group succeeded in growing a new ferromagnetic DMS, (Ga,Mn)As [7].

This paper reviews the recent advances of III-V DMSs, especially (Ga,Mn)As, and is organized in the following way. Section 2 describes the MBE growth of $(\mathrm{Ga}, \mathrm{Mn}) \mathrm{As}$ and the basic properties of the grown films. The magnetic and magnetotransport properties $(\mathrm{Ga}, \mathrm{Mn})$ As are presented in Sec. 3, together with the discussion of the possible origin of ferromagnetism. Properties of ( $\mathrm{Ga}, \mathrm{Mn})$ As heterostructures are presented in Sec. 4.

\section{Molecular beam epitaxial growth}

In order to make non-magnetic semiconductor magnetic, one needs to introduce a high concentration of magnetic elements. At the beginning of III-V DMS research, it was not obvious whether one can introduce magnetic elements such as $\mathrm{Mn}$ into host III-V compound to a few percent or more, because the solid solubility of such elements in III-V compounds is known to be low $\left(<10^{19} \mathrm{~cm}^{-3}\right)$. Segregation of impurities during MBE growth was another difficulty in obtaining high concentration of magnetic elements. These difficulties were overcome by using low growth temperatures.

All the layers described in this paper were grown by solid source MBE using elemental sources $\mathrm{Ga}, \mathrm{Mn}$, and As. No intentional doping was used, and all growth was carried out under the As stabilized condition. (Ga,Mn)As films were grown on semi-insulating (001) GaAs substrates at a growth rate of $0.6 \mu \mathrm{m} / \mathrm{h}$. Mn content $x$ in the $\left(\mathrm{Ga}_{1-x} \mathrm{Mn}_{x}\right)$ As films was varied from 0.015 to 0.071 , which was calibrated by electron probe micro-analysis (EPMA) measurement results on (In,Mn)As layers. Reflection high energy electron diffraction (RHEED) patterns were used to monitor the surface reconstruction during the growth. A typical thin film structure consists of a 150-200 nm (Ga,Mn)As layer (grown at a substrate temperature $\left.T_{\mathrm{s}}=250^{\circ} \mathrm{C}\right) /$ a $100 \mathrm{~nm}$ low-temperature GaAs layer $\left(T_{\mathrm{s}}=250^{\circ} \mathrm{C}\right)$ 
or a $100 \mathrm{~nm}$ high temperature AlGaAs buffer layer $\left(T_{\mathrm{s}}=700^{\circ} \mathrm{C}\right) /$ a $100 \mathrm{~nm} \mathrm{GaAs}$ buffer layer $\left(T_{\mathrm{s}}=700^{\circ} \mathrm{C}\right)$. When the low-temperature GaAs buffer layer growth was initiated at $250^{\circ} \mathrm{C}$, the $c(4 \times 4)$ reconstruction pattern of $\mathrm{GaAs}$ changed to a $(1 \times 1)$ pattern. No change in the beam fluxes from the high-temperature growth of GaAs was made for this low-temperature GaAs growth. The (Ga,Mn)As growth was started by simply opening the Mn shutter during the low-temperature GaAs growth, keeping the substrate temperature at the same $250^{\circ} \mathrm{C}$. No special precaution was taken at the start of $(\mathrm{Ga}, \mathrm{Mn})$ As growth. Although the properties of grown ( $\mathrm{Ga}, \mathrm{Mn}) \mathrm{As}$ do depend on growth parameters such as As overpressure and $T_{\mathbf{s}}$, as long as one follows the established growth procedure, the properties of ( $\mathrm{Ga}, \mathrm{Mn})$ As films were reproducible; for example, for a given Mn concentration $x$, the ferromagnetic transition temperature $T_{\mathrm{C}}$ was always about $2000 x \pm 10 \mathrm{~K}$. The surface reconstruction of $(\mathrm{Ga}, \mathrm{Mn})$ As was $(1 \times 2)$ during and after the growth. If the $\mathrm{Mn}$ flux and/or the substrate temperature was too high, a complicated RHEED pattern appeared, indicating the appearance of the MnAs (NiAs-structure) second phase on the surface. The details of the growth are described in Refs. [7-9]. Clear RHEED oscillations were observed at the initial growth stage (even without $\mathrm{Mn}$ ), which indicate the growth mode being two-dimensional. Results on RHEED oscillation of GaAs at low temperature was reported elsewhere [10]. Using low-temperature MBE, (In, Mn)As dots have also been grown recently on GaAs substrates [11].

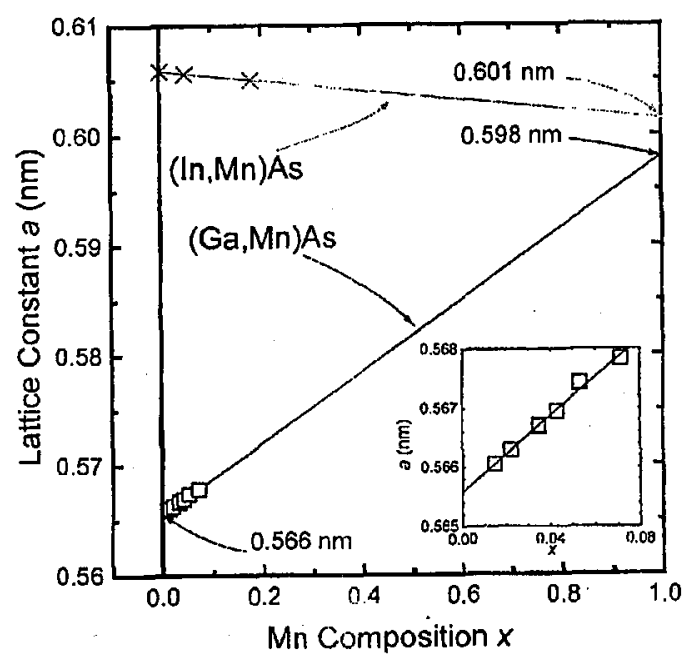

Fig. 1. Lattice constant $a$ vs. Mn composition $x$ in (Ga,Mn)As films and in (In,Mn)As films. $a$ was determined by X-ray diffraction at room temperature.

Figure 1 shows the lattice constants of the grown $(\mathrm{Ga}, \mathrm{Mn})$ As layers determined by X-ray diffraction together with the lattice constant of (In, Mn)As reported earlier [5]. The lattice constant, $a$, was calculated from the diffraction peak position in X-ray diffraction patterns assuming that $(\mathrm{Ga}, \mathrm{Mn})$ As was fully strained and that $(\mathrm{Ga}, \mathrm{Mn}) \mathrm{As}$ had the same elastic constant as GaAs. The assumption that 
( $\mathrm{Ga}, \mathrm{Mn})$ As is fully strained on GaAs was confirmed by asymmetric X-ray diffraction by using a double crystal diffractometer with (115) reflection. As can be seen from Fig. 1, $a$ increases linearly with $x$ up to 0.071 , above which segregation of Mn occurs even at low growth temperatures. The extrapolated lattice constants for GaAs $(0.566 \mathrm{~nm})$ and $\mathrm{MnAs}(0.598 \mathrm{~nm})$ are in good agreement with the known GaAs lattice constant $(0.56533 \mathrm{~nm})$ and with the MnAs lattice constant extrapolated from the (In, Mn)As side $(0.601 \mathrm{~nm})$ [5]. This agreement suggests that all the $\mathrm{Mn}$ atoms were incorporated in the zinc-blende alloy. It was recently reported by Shioda et al. that $\mathrm{Mn}$ is indeed substitutionally incorporated in to the Ga sublattice by extended X-ray absorption fine structure measurement [12].

\section{Magnetic and magnetotransport properties, and the origin of ferromagnetism}

The presence of ferromagnetic order in (Ga,Mn)As films at low temperature was revealed by magnetization measurements using a SQUID magnetometer. Figure 2 shows the magnetic field dependence of the magnetization of a sample with $x=0.035$ at $5 \mathrm{~K}$. The magnetic field $B$ was applied parallel to the epitaxial film (perpendicular to the growth direction). The sharp, square hysteresis in Fig. 2 indicates a well-ordered ferromagnetic structure in the film. The inset in Fig. 2 shows the temperature dependence of the remanence of the same film, where a clear transition to the ordered state at $60 \mathrm{~K}$ is seen.

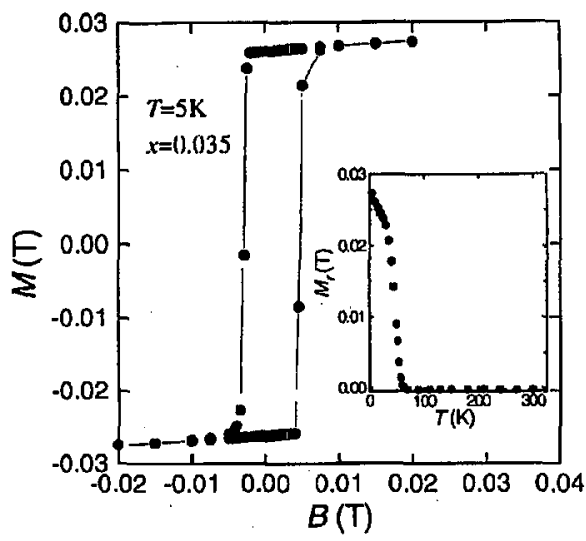

Fig. 2. Magnetic field dependence of magnetization $M$ at $5 \mathrm{~K}$ for a $(\mathrm{Ga}, \mathrm{Mn})$ As film with Mn content $x=0.035$. The field was applied parallel to the sample surface. The inset shows the temperature dependence of the remanent magnetization $M_{\mathrm{r}}$ of the same sample.

Magnetization of the ( $\mathrm{Ga}, \mathrm{Mn})$ As film shown in Fig. 2 gradually increases at higher fields and then saturates at the saturation magnetization $M_{\mathrm{s}}=0.040 \mathrm{~T}$. Most of the samples showed this paramagnetic response at high fields, which seems to be correlated to the transport properties of the films; the metallic samples show only small contribution of the paramagnetic component, whereas the contribution 
can be comparable to the ferromagnetic component in insulating samples. $M_{\mathrm{s}}$ of the $(\mathrm{Ga}, \mathrm{Mn})$ As film is given by $M_{\mathrm{Mn}}=N_{\mathrm{Mn}} g \mu_{\mathrm{B}} S_{\mathrm{Mn}}$, where $N_{\mathrm{Mn}}\left(=7.7 \times 10^{20} \mathrm{~cm}^{-3}\right.$ for $x=0.035)$ is the nominal Mn concentration, $g=2$ the $g$-factor of $\mathrm{Mn}, \mu_{\mathrm{B}}$ is the Bohr magneton, and $S$ the spin of $\mathrm{Mn}$. The possible values for $S$ of $\mathrm{Mn}$ are $5 / 2$ for $\mathrm{Mn}^{2+}$ and $4 / 2$ for $\mathrm{Mn}^{3+} . M_{\mathrm{s}}=0.040 \mathrm{~T}$ gives $S=2.2$, in between the two. Although it is difficult to determine $S$ from these experiments alone because of the possible presence of error involved in determining $x$ and the nonuniformity of $x$ over the sample, low-temperature high-field saturation was always consistent with the fully aligned $\mathrm{Mn}$ spin of $5 / 2$ assuming nominal $x$. Absence of remanence above $75 \mathrm{~K}$ in the inset of Fig. 2 confirms that the concentration of $\operatorname{MnAs}\left(T_{\mathrm{C}}=310 \mathrm{~K}\right)$ is extremely low.

The rotation of magnetic field revealed that the easy-axis is in-plane. This is in contrast to what has been reported for ( $\mathrm{In}, \mathrm{Mn}) \mathrm{As}$ films, where perpendicular easy-axis was observed [13]. This difference may be explained by the magneto-elastic effect; the $(\mathrm{In}, \mathrm{Mn})$ As layers were under biaxial tensile strain, whereas the present $(\mathrm{Ga}, \mathrm{Mn})$ As layers were under compressive strain. The $(\mathrm{Ga}, \mathrm{Mn})$ As layers grown on a (In,Ga)As buffer layer to introduce biaxial tensile stress exhibited the perpendicular easy-axis, supporting the present explanation [14].

Sheet resistance $R_{\text {sheet }}$ and Hall resistance $R_{\text {Hall }}$ of $200 \mathrm{~nm}$ (Ga,Mn)As layers $(x=0.015 \div 0.071)$ grown on $(\mathrm{Al}, \mathrm{Ga})$ As buffer layer $/(001) \mathrm{GaAs}$ substrates were measured using a standard dc transport measurement setup in the temperature range of $2-300 \mathrm{~K}$ with magnetic fields $B$ up to $12 \mathrm{~T}$. Temperature dependence of $R_{\text {sheet }}$ of samples with intermediate Mn composition $x(0.035-0.053)$ showed that they were on the metal side of the metal-insulator transition, whereas low and high $x$ samples were on the insulator side. To avoid complication arising from the localization effects, we hereafter concentrate on the metallic samples especially the one with $x=0.053$; results for other metallic samples were essentially the same.

$R_{\text {Hall }}$ can be expressed as

$$
R_{\text {Hall }}=\frac{R_{0}}{d} B+\frac{R_{S}}{d} M
$$

where $R_{0}$ is the ordinary (normal) Hall coefficient, $R_{S}$ the anomalous Hall coefficient, $d$ the sample thickness, $M$ the magnetization of the sample. $R_{S}$ can be shown to be proportional to $R_{\text {sheet }}$ in the present samples and thus $R_{S} / d=c R_{\text {sheet }}$, where $c$ is a constant, indicating that the skew scattering is responsible for the anomalous Hall effect [15]. Since the anomalous Hall term is the dominant term, one can determine $M$ of the sample from $R_{\mathrm{Hall}}$. In order to determine the conduction type and the carrier concentration, one has to measure the ordinary Hall coefficient as a slope of the $R_{\text {Hall }}-B$ curve at low temperature under high magnetic field, where $M$ is saturated and constant.

The results of the magnetotransport measurements on a sample with $x=0.053$ are shown in Fig. 3. Reflecting the dominant contribution of the anomalous Hall term on $R_{\mathrm{Hall}}$, the temperature and magnetic field dependence of $R_{\mathrm{Hall}}$ is approximately proportional to that of the magnetization. As temperature $T$ decreases, the sheet resistivity $R_{\text {sheet }}$ first increases along with the negative magnetoresistance. The zero-field resistivity peaks at around $T_{\mathrm{C}}$ and then starts to 
(a)

(b)

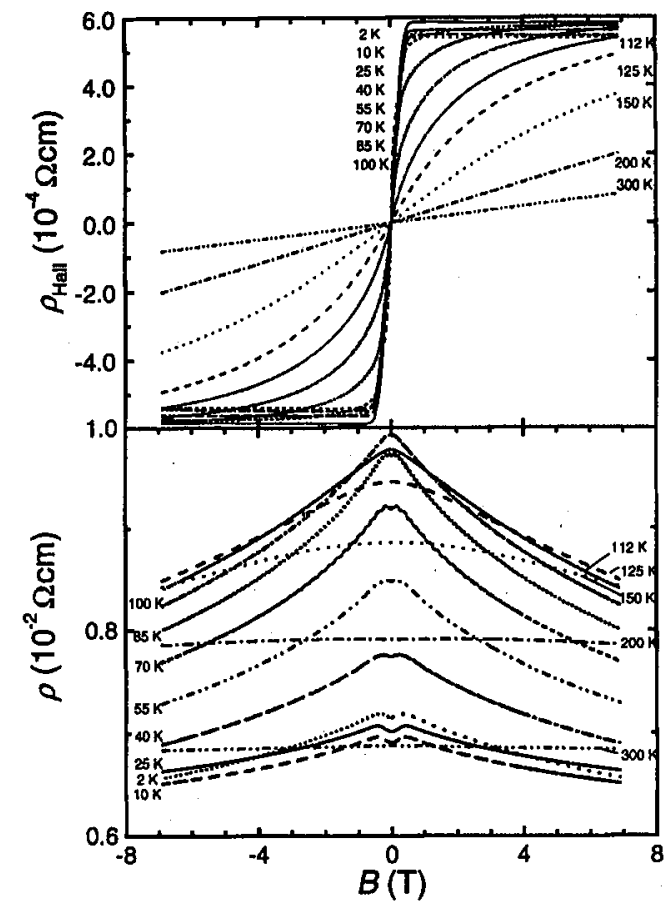

Fig. 3. Magnetic field dependence of Hall resistivity $\rho_{\mathrm{Hall}}$ (a) and resistivity $\rho$ (b) of $(\mathrm{Ga}, \mathrm{Mn})$ As with temperature as a parameter.

decrease. The negative magnetoresistance decreases as well. Using Arrot plots, where $\left(R_{\text {Hall }} / R_{\text {sheet }}\right)^{2}$ is plotted against $\left(B /\left(R_{\text {Hall }} / R_{\text {sheet }}\right)\right.$ ) (not shown), one can obtain the temperature dependence of the saturation magnetization $M_{\mathrm{s}}$ and Curie temperature $T_{\mathrm{C}}$; for the present sample $T_{\mathrm{C}}=110 \mathrm{~K}$. The temperature dependence of $M_{\mathrm{s}}$ can be fitted with that calculated from a Brillouin function, i.e., ferromagnetism observed in ( $\mathrm{Ga}, \mathrm{Mn}$ )As can be understood in the framework of a simple mean field theory. The paramagnetic Curie temperature $\theta$ was obtained from the temperature dependence of the inverse of the zero-field slope of $R_{\text {Hall }} / R_{\text {sheet }}$ (proportional to susceptibility $\chi$ ); a straight line characteristic of Curie-Weiss law was obtained. For all the samples, $\theta$ was close to $T_{\mathrm{C}}$. The slope of $R_{\mathrm{Hall}}-B$ measured at $10 \mathrm{~K}$ revealed that the conduction type was $p$-type with a hole concentration of $1.0 \times 10^{20} \mathrm{~cm}^{-3}$.

The $R_{\text {sheet }}-T$ curve showed a maximum at around $T_{\mathrm{C}}$, which moved to higher temperatures with increasing $B$, as shown in Fig. 4. This behavior of $R_{\text {sheet }}$ is believed to be due to the scattering of carriers by magnetic fluctuation via exchange interaction (critical scattering), which has been observed in magnetic semiconductors [16], as opposed to metal-insulator transition proposed by Van Esch et al. [17]. This interpretation is supported by the fact that the temperature dependence of the high-temperature part is proportional to $\chi T$, as expected from the critical scattering. The observed negative resistance can be understood as the 


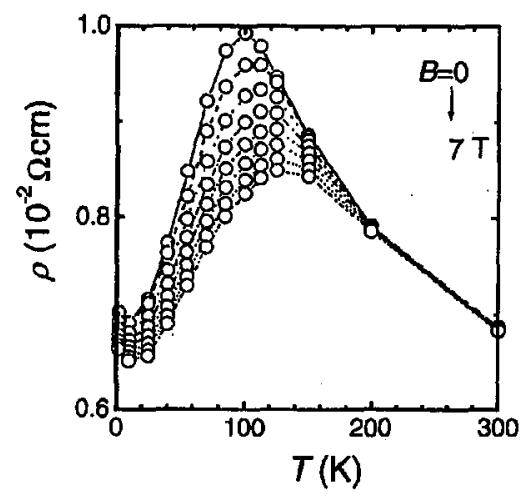

Fig. 4. Temperature dependence of sheet resistivity $\rho$ of the sample shown in Fig. 3. Critical behavior of $\rho$ is clearly observed together with negative magnetoresistance in all the temperature range investigated.

reduction of scattering by aligning the spins by $B$. Well above $T_{\mathrm{C}}$, one can fit. the $B$ dependence of $R_{\text {sheet }}$ to the following critical scattering resistivity formula:

$$
\rho_{s}=2 \pi^{2} \frac{k_{\mathrm{F}}}{n e^{2}} \frac{m^{2} \Gamma^{2}}{h^{3}} n_{s}\left[S(S+1)-\langle S\rangle^{2}\right],
$$

where $k_{\mathrm{F}}$ is the Fermi wave vector, $n$ the hole concentration, $m$ the effective mass, $\Gamma$ the $p-d$ exchange, $n_{s}$ the Mn concentration, $S=5 / 2$, and $\langle\boldsymbol{S}\rangle$ the average spin on $\mathrm{Mn}$ [18]. Other symbols have their usual meanings. By using the measured hole concentration and the effective mass of $0.5 m_{0}$ ( $m_{0}$ the free electron mass), the fit of Eq. (2) to experimental results yields $\Gamma=150 \pm 40 \mathrm{eV} \AA^{3}$ or $N_{0} \beta \approx 3.3 \mathrm{eV}$ in terms of $N_{0} \beta$ commonly used to describe $p-d$ interaction in DMSs. Essentially the same exchange was obtained from other metallic $(\mathrm{Ga}, \mathrm{Mn}) \mathrm{As}$ samples. This rather large $\Gamma$ should be compared to the typical $p-d$ exchange in II-VI based DMSs which is $1 \mathrm{eV}$ at most. Although the origin of this enhancement is not clear at the moment, similar $p-d$ exchange in GaAs doped with $\mathrm{Mn}$ was reported by Szczytko et al. [19], who investigated magneto-optical properties of Mn doped GaAs.

Since the magnetic interaction between $\mathrm{Mn}$ has been shown to be antiferromagnetic in semi-insulating fully compensated ( $\mathrm{Ga}, \mathrm{Mn}) \mathrm{As}$ using $\mathrm{Sn}$ as a donor [20], the ferromagnetic interaction in (Ga,Mn)As is most likely hole induced. We have examined whether the ferromagnetism of $(\mathrm{Ga}, \mathrm{Mn}) \mathrm{As}$ fits into the framework of Ruderman-Kittel-Kasuya-Yosida (RKKY) interaction, which was shown to be responsible for the carrier induced ferromagnetism in a IV-VI compound $\mathrm{PbSnMnTe}$ [4]. The calculated $T_{\mathrm{C}}$ using the exchange constant and the hole concentration determined experimentally was in good agreement with the experimentally determined $T_{\mathrm{C}}$ [21], although the result depends on the cut-off length of the RKKY interaction, i.e., the mean free path. Because of this quantitative agreement, the RKKY interaction is most likely responsible for the appearance of ferromagnetism in $(\mathrm{Ga}, \mathrm{Mn})$ As. 


\section{Heterostructures}

In order to further examine the carrier mediated magnetic interaction, we have grown $(\mathrm{Ga}, \mathrm{Mn}) \mathrm{As}$ based heterostructures and measured the magnetic interaction between the layers. High quality $(\mathrm{Ga}, \mathrm{Mn})$ As superlattice structures of $24 \mathrm{~nm}$ period $(12 \mathrm{~nm}(\mathrm{Ga}, \mathrm{Mn})$ As and $12 \mathrm{~nm} \mathrm{GaAs})$ were demonstrated and shown to exhibit ferromagnetism [22]. ( $\mathrm{Ga}, \mathrm{Mn})$ As quantum wells were also realized [23]. We have grown a series of structures with a $30 \mathrm{~nm}(\mathrm{Ga}, \mathrm{Mn}) \mathrm{As}(x=0.04)$ layer and a $30 \mathrm{~nm}(\mathrm{Ga}, \mathrm{Mn}) \mathrm{As}(x=0.02)$ layer separated by a nonmagnetic $(\mathrm{Al}, \mathrm{Ga}) \mathrm{As}$ layer and examined the magnetic coupling between the two magnetic layers. The thickness of the intermediary layer was fixed to 10 monolayers and the $\mathrm{Al}$ composition was varied $\left(x_{\mathrm{Al}}=0.16,0.29\right)$, hence the barrier in the valence band. Magnetic measurements ( $M-B$ curves, $B$ applied in the plane) revealed that the two layers were magnetically decoupled and the $M-B$ curve was a simple addition of the two individual $M-B$ curves measured on separately grown samples for $x_{\mathrm{Al}}=0.29$. On the other hand, for $x_{\mathrm{Al}}=0.16$, the magnetization curve showed only one step, indicating that the two magnetic layers were now ferromagnetically coupled. The magnetic coupling between the two ferromagnetic $(\mathrm{Ga}, \mathrm{Mn})$ As films separated by a nonmagnetic GaAs layer was also shown to be a function of thickness of the intermediary GaAs layer [24]. Both sets of results indicate the critical role of the holes in the intermediary layer on the magnetic coupling. This is consistent with the RKKY interaction being the origin of the magnetic coupling in the present material system.

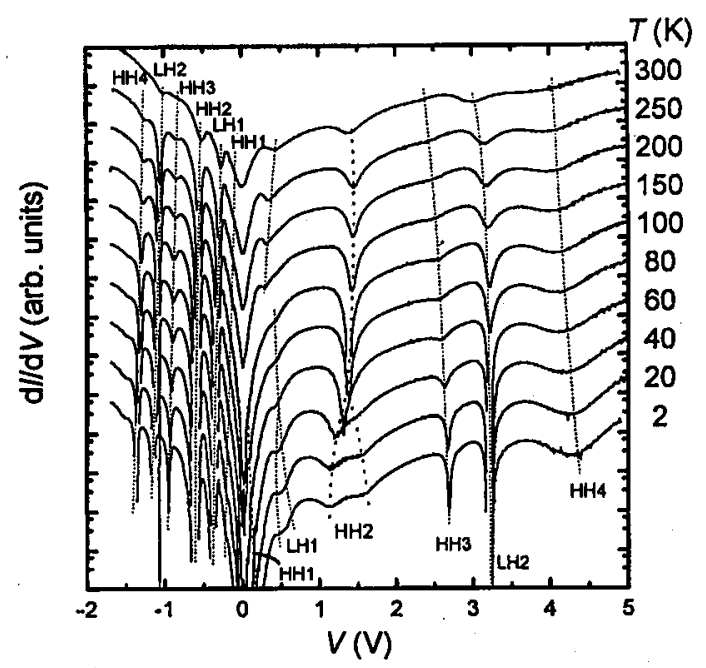

Fig. 5. $\mathrm{d} I / \mathrm{d} V-V$ curves at various temperatures. Positive bias corresponds to the hole injection from the $(\mathrm{Ga}, \mathrm{Mn})$ As side.

Figure 5 shows conductance versus applied voltage curves of an AlAs/GaAs/ AlAs ( $5 \mathrm{~nm}$ each) double barrier resonant tunneling diode with ferromagnetic $p$-type $(\mathrm{Ga}, \mathrm{Mn}) \mathrm{As}$ on one side and $p$-type GaAs on the other. It exhibited a spon- 
taneous resonant peak splitting when holes were injected from the (Ga,Mn)As side. The spontaneous resonant peak splitting, without magnetic field, started to develop below the ferromagnetic transition temperature of (Ga,Mn)As. The magnitude of the splitting can be described by a Brillouin function showing the origin of peak splitting being the spin splitting in the valence band of ferromagnetic $(\mathrm{Ga}, \mathrm{Mn})$ As [25].

\section{Conclusion}

In conclusion, we have prepared a new III-V DMS, (Ga,Mn)As, and its related heterostructures by $\mathrm{MBE}$. X-ray diffraction measurements showed that the lattice constant of the $(\mathrm{Ga}, \mathrm{Mn})$ As films increases linearly with $\mathrm{Mn}$ composition, suggesting homogeneous incorporation of $\mathrm{Mn}$ atoms in the zinc-blende alloy. Magnetization of the ( $\mathrm{Ga}, \mathrm{Mn}$ )As films was determined from magnetic as well as magnetotransport measurements. The highest $T_{\mathrm{C}}$ so far obtained is $110 \mathrm{~K}$. From the spin-disorder scattering, the exchange interaction was determined to be $3 \mathrm{eV}$, which was shown to be consistent with the value expected from the $T_{\mathrm{C}}$ based on the RKKY interaction. The magnetic interaction between the two ferromagnetic (Ga,Mn)As layers was shown to be a function of intermediary non-magnetic $(\mathrm{Al}, \mathrm{Ga}) \mathrm{As}$ layers in between the $(\mathrm{Ga}, \mathrm{Mn})$ As layers. Resonant tunneling diodes with a $(\mathrm{Ga}, \mathrm{Mn}) \mathrm{As}$ emitter exhibited a spontaneous splitting in conductance versus voltage curve. The new III-V based DMSs are believed to open up a new field in semiconductor technology, where both semiconducting properties and magnetic properties play critical roles.

\section{Acknowledgments}

It is my pleasure to acknowledge valuable and fruitful collaboration with F. Matsukura, A. Shen, N. Akiba, Y. Sugawara, and T. Kuroiwa of Tohoku University and Y. Iye, S. Katsumoto, and A. Oiwa of the University of Tokyo. This work was partially supported by a Grant-in-Aid for Scientific Research Priority Area "Spin Controlled Semiconductor Nanostructures" (No. 09244103) from the Ministry of Education, Science, Sports and Culture, Japan, and by the "Research for the Future" Program (No. JSPS-RFTF97P00202) from the Japan Society for the Promotion of Science.

\section{References}

[1] T. Kasuya, A. Yanase, Rev. Mod. Phys. 40, 684 (1968).

[2] Semiconductors and Semimetals, Eds. J,K. Furdyna, J. Kossut, Vol. 25, Academic, New York 1988.

[3] A. Haury, A. Wasiela, A. Arnoult, J. Cibert, S. Tatarenko, T. Dietl, Y. Merle d'Aubigné, Phys. Rev. Lett. 79, 511 (1997).

[4] T. Story, R.R. Gałązka, R.B. Frankel, P.A. Wolff, Phys. Rev. Lett. 56, 777 (1986).

[5] H. Munekata, H. Ohno, S. von Molnár, A. Segmüller, L.L. Chang, L. Esaki, Phys. Rev. Lett. 63, 1849 (1989).

[6] H. Ohno, H. Munekata, T. Penney, S. von Molnár, L.L. Chang, Phys. Rev. Lett. 68,2664 (1992). 
[7] H. Ohno, A. Shen, F. Matsukura, A. Oiwa, A. Endo, S. Katsumoto, Y. Iye, Appl. Phys. Lett. 69, 363 (1996).

[8] F. Matsukura, A. Oiwa, A. Shen, Y. Sugawara, N. Akiba, T. Kuroiwa, H. Ohno, A. Endo, S. Katsumoto, Y. Iye, Appl. Surf. Sci. 113/114, 178 (1997).

[9] A. Shen, H. Ohno, F. Matsukura, Y. Sugawara, N. Akiba, T. Kuroiwa, A. Oiwa, A. Endo, S. Katsumoto, Y. Iye, J. Cryst. Growth 175/176, 1069 (1997).

[10] A. Shen, Y. Horikoshi, H. Ohno, S.P. Guo, Appl. Phys. Lett. 71, 1540 (1997).

[11] S.P. Guo, H. Ohno, A. Shen, F. Matsukura, Y. Ohno, Appl. Surf. Sci. 130-132, 797 (1998).

[12] R. Shioda, K. Ando, T. Hayashi, M. Tanaka, Phys. Rev. B 53, 1100 (1998).

[13] H. Munekata, A. Zaslavsky, P. Fumagalli, R.J. Gambino, Appl. Phys. Lett. 63, 2929 (1993).

[14] H. Ohno, F. Matsukura, A. Shen, Y. Sugawara, A. Oiwa, A. Endo, S. Katsumoto, Y. Iye, in: Proc. 23rd Int. Conf. on Physics of Semiconductors, Eds. M. Scheffler, R. Zimmermann, World Scientific, Singapore 1996, p. 405.

[15] The Hall Effect and its Applications, Eds. C.L. Chien, C.W. Westgate, Plenum, New York 1980, p. 43.

[16] S. von Molnár, T. Kasuya, Phys. Rev. Lett. 21, 1757 (1968).

[17] A. Van Esch, L. Van Bockstal, J. De Boeck, G. Verbanck, A.S. van Steenbergen, P.J. Wellmann, B. Grietens, R. Bogaerts, F. Herlach, G. Borghs, Phys. Rev. B 56, 13103 (1997).

[18] T. Kasuya, Prog. Theor. Phys. 16, 45 (1956).

[19] J. Szczytko, W. Mac, A. Stachow, A. Twardowski, P. Becla, J. Tworzydlo, Solid State Commun. 99, 927 (1996).

[20] Y. Satoh, N. Inoue, Y. Nishikawa, J. Yoshino, in: 3rd. Symp. on Physics and Application of Spin Related Phenomena in Semiconductors, Sendai (Japan) 1997, Eds. H. Ohno, Y. Oka, J. Yoshino, Sendai (Japan) 1997, p. 23.

[21] F. Matsukura, H. Ohno, A. Shen, Y. Sugawara, Phys. Rev. B 57, R2037 (1998).

[22] A. Shen, H. Ohno, F. Matsukura, Y. Sugawara, Y. Ohno, N. Akiba, T. Kuroiwa, Jpn. J. Appl. Phys. 36, L73 (1997).

[23] T. Hayashi, M. Tanaka, K. Seto, T. Nishinaga, K. Ando, Appl. Phys. Lett. 71, 1825 (1997).

[24] N. Akiba, F. Matsukura, A. Shen, Y. Ohno, H. Ohno, A. Oiwa, S. Katsumoto, Y. Iye, to be published in Appl. Phys. Lett.

[25] H. Ohno, N. Akiba, A. Shen, K. Ohtani, F. Matsukura, Y. Ohno, Appl. Phys. Lett 73, 363 (1998). 